\title{
Spatio-temporal Suspension and Imagery in Popular Music Recordings
}

\author{
Peter Long \\ Western Sydney University \\ p.long@westernsydney.edu.au
}

\begin{abstract}
Constructed spatiality and perceived imagery in popular music recordings has been in evidence since the 1930 s as "a fundamentally pictorial tradition (...) reverb and echo effects deployed in combination with certain lyrics to render aural vistas" (Doyle, 2004: 32). Spatiality underwent a transformation in the 1950s with echo and reverberation increasingly employed as timbral and rhythmic enhancement to music. By the 1960s, with advances in recording technologies and the emergence of psychedelic music, sound environments openly challenged notions of spatial reality, providing a perceptual bridge between auditory and visual realms. This practice-led enquiry focuses upon the experience of spatio-temporal suspension in popular music recordings. Through praxical engagement with consumer audio technologies, it aims to define a timeless, suspended aesthetic in music and sound, by drawing on neuroscientific research into dynamic forms of vitality in the time-based arts (Stern, 2010) and demonstrating the multimodal nature of these recordings.
\end{abstract}

KEYWORDS: spatiality, affect, suspension, echo, reverberation, perception

The composition that is part of this study can be found here:

http://www.iaspmjournal.net/index.php/IASPM_Journal/article/downloadSuppFile/821/240

\section{Background}

This investigation examines the phenomenon of spatio-temporal suspension and its aesthetic application in popular music recordings. It derives inspiration from sound environments constructed through experimental studio and performance techniques developed in the mid-1960s, in particular psychedelic rock. Many of the techniques developed were born of attempts to represent sonically altered states of consciousness. Reising (2009) observes psychedelic music's preoccupation with 
perceptions of time and space emanated from artist's attempts to express the disorienting effects of LSD in musical terms, including:

the aural synesthesia in which sounds seem to burst into vibrant colors (...) the wash of sight and sound in which it is difficult to distinguish foreground from background (...) and the elasticity of space and time which expand and contract seemingly to fit the mood or aura of the environment. (Reising 2009: 524).

The apparent distortion of space and time in recordings of this period were expressed via studio effects that appeared to bend or stretch time and spatial dimensions. The use of spatiality in sound recordings and the ability to conjure imagery however, dates to the 1920s. Doyle (2004) observes a "fundamentally pictorial tradition (...) reverb and echo effects deployed in combination with certain lyrics to render aural vistas" (2004: 32). By the 1940s the aesthetic use of reverberation in sound recording would be used to denote twilight settings, indicating shifts from "consciousness to dream, from the realm of the ego to the $\mathrm{id}^{\text {" }}$ (2004: 36). The potential of reverberation and echo to elicit dream-like spaces, coupled with the temporally plastic medium of audiotape became important tools at the disposal of producers of psychedelic music in the mid-1960s. But how do these recordings elicit imagery and contribute to a sense of temporal suspension?

\section{Theoretical observations}

Ihde (2007) suggests that vision and sound tend to be regarded as separate or atomised, but there are often instances where they overlap (2007: 49). Noting that the auditory threshold is usually regarded as silence, he presupposes that night would be the threshold for vision. In observations of wind however, Ihde notes that the passage of air itself cannot be seen, only its visual and auditory effects, which involve perceptions of movement. In the visual realm, the static object is effectively mute and auditory objects rendered invisible unless movement signals their presence. Although sound often serves as "anticipatory clues for ultimate visual fulfillments" more importantly we hear shapes in sound, using the example of a child's game where an object is rolled around inside a closed box and a participant is invited to guess its identity (2007: 54, 61-62). The auditory information contained in the texture, composition and shape-aspect of an object is rich and complex, and although abstracted from its source, is heavily informed by learned visual associations. Schaeffer (2004) also recognised the "richness of this perception" in defining the sonorous object, noting that "much of what we thought was heard was in reality only seen, and explicated, through (...) context" (2004: 78).

Stern (2010) suggests that vitality affects - neurological waves of response to internal and external stimuli - set down pathways for perceptions of movement in space and time. As the temporal and dynamic manifestations of movement trigger neurotransmitters associated with autonomic nervous systems, affects are mapped onto consciousness and perception from birth, informing how humans are attuned to aesthetic waveforms; we mirror previously learned movement and experience (2010: 42). Stern observes that "cross-modal merging and synesthesias are initially more common in infancy, and are in fact the norm" (2010: 35). In the time-based arts especially, Stern observes the capacity for vitality forms to "elicit similar felt states regardless of what modality they arise from" (2010: 76) giving rise to collaborations across art forms or correspondences: 


\begin{abstract}
"Correspondences" between art forms are necessarily created because of the meta-modal nature of vitality forms that assure a common ability to render similar, but not identical, experiences. The magic lies in pairing the similar with the "not exactly the same". (Stern 2010: 78).
\end{abstract}

This potential for correspondence across art forms suggests sensorial pairing of the moving image with the auditory and musical, given the "meta-modality and potential speed of modulation" of vitality affects (2010: 79). Bearing in mind Ihde's observations, this suggests cognitive mapping occurring in early development has the potential to imprint across the sensory modes in a favorable environment.

\title{
Affect in psychedelic music
}

Sound environments presented in psychedelic music are especially conducive to imagery as the studio techniques employed will often abstract sounds and instruments contextually from their source, without entirely losing identity. Clarke (2005) observes that when sounds are presented "in a transformed, or radically decontextualised fashion" attention is drawn instinctively to "the structure of the stimulus information" (2005: 33). When John Lennon sings "let me take you down, cause I'm going to" just before the 1:00 minute edit point of "Strawberry Fields Forever" (Parlophone, 1967), the odd vocal timbre resulting from the change in tape speed combined with an abrupt switch of instrumentation contributes to the sense of stepping into a space where "nothing is real". Distortion of time and space by speed manipulation abounds in early psychedelic music recordings, from the surreal sequence at 0.50 in Pink Floyd's "See Emily Play" (Columbia Records, 1967) to the extraterrestrial worlds elicited in the tape echo introduction to Jimi Hendrix's "... And The Gods Made Love" from Electric Ladyland (Track Records, 1968). Temporality is transformed not only by varying tape speed but also in utilising flanging and reversal in a disorienting yet exhilarating manipulation of time and space. These techniques are used to this day; Radiohead's "Daydreaming" from $A$ Moon Shaped Pool (XL Recordings, 2016) exploits the analog capabilities of magnetic tape with a lurching varispeed introduction and instances of backwards and time-stretched vocals throughout, contributing to a somnambulistic, floating netherworld.

However not all such distortions of time and space are derived from studio techniques alone; many are musical in nature. Drone and repetition are common in psychedelic music, with McClary (2004) observing the 20th century's preoccupation with cyclic repetition, tracing a lineage stretching from Stravinsky through to Riley, Reich and Glass, the Beatles, Led Zeppelin and eventually Beck (2004: 289). The influence of Indian music upon both the minimalists and psychedelic music is well documented, and the use of drone as both a musical and timbral device became a defining feature of psychedelic rock following The Yardbird's "Heart Full Of Soul" (1965) and The Kinks' "See My Friends" (1965). The sense of temporal suspension resulting from repetition and an emphasis on timbre also became a blueprint for Brian Eno's development of ambient music in the 1970s, and Eno's vertical approach to sound and observance of imagery in music informs the methodology of this project. 


\section{Eno as methodology}

I want to make a kind of music that had the long "now" and the "big" here, and for me that meant this idea of expanding the music out to the horizons (...) this is the sense of making the 'now' longer. (Ward and Cardazzo 1989: 4.19).

Eno refers to music in temporal and spatial terms, using terminology normally ascribed to the visual senses. When Imaginary Landscapes was made in 1989, he had already designed several video installations and envisioned sound in terms of imagined spaces. Achtermann (2016) positions this as "music outside of time" conceiving of Eno's oeuvre as "constructs of the imagination" and importantly observes Eno's ambient soundscapes do not imitate nature, but recreate "the sense of some existing environment by imitating processes and systems of the natural world" (2016: 92-96). From observations of natural processes such as wave phenomena, comparisons can be made with way Eno's music evolves in a continual unfolding of auditory and musical events, contributing to "a sense of time slowed, even timelessness" (2016: 96). The indeterminacy of this generative approach or aesthetic of time also reveals a gradual removal of authorial identity, demonstrated on Discreet Music (1975) as the pieces generate multiple, unending variation, enacting Eno's mantra "repetition is a form of change" (Scoates 2013: 36).

From a series of practice-led experiments I have distilled characteristics from the music of Eno and others that appear to contribute to the idea of suspension in popular music and have employed many of these in the composition of the piece "Submarine Bells" (2016).

\section{Suspension characteristics}

A few sonic characteristics are employed within popular music to lead to a sense of suspension. A constancy of process is used, evolving through apparent stasis. Although appearing to lack movement, internal processes evolve constantly, characterised by use of repeated arpeggios and motifs, drones and grounds. The music may aim for harmonic stasis, using modal or quartal/quintal harmony, and the avoidance of functional cadential harmony or any sense of resolution. Rhythmic figures are ambiguous and/or static, obfuscating meter, creating music that has no apparent pulse. There is an ambiguity of tonality, using metallophones, percussion and other instruments that exhibit degrees of inharmonicity, such as bells and cymbals. Extended and non-standard instrumental techniques, are employed to produce unusual sounds and timbres. Instrumentation adopts sounds that fall outside standard western instrumentation, often focused on an ethnographic soundworld. The sound medium is manipulated to distort, diffuse or otherwise alter the characteristics of instruments and sounds. This includes reversal of the audio signal to alter envelope characteristics and speeding or slowing to contract or extend time. A sense of overt/unusual spatial awareness is sought, an exaggerated or odd juxtaposition of auditory spaces, reinforced through reverberation and echo, including conspicuous panning to imply movement. These techniques are used texturally to obfuscate or blur signals and involve a degree of abstraction. These techniques involve a degree of abstraction from an auditory source, with a focus upon the phenomena generated. Shouse (2005) notes that "it is affect's 
'abstractivity' that makes it transmittable in ways that feelings and emotions are not" (2005: para. 15).

"Submarine Bells" makes use of commonly available consumer technologies, improvised on an iPad utilizing the Loopy HD app (Version 1.6.2, 2016) to record unsynchronized and differently timed audio loops in an echo of Eno's generative approach. It employs a basic yet effective array of reverberation, analog delays and panning to create atypical spatial effects. Harmonically, the piece is based on a tone cluster comprising intervals of a fifth (GDAE) to impart tonal ambiguity. Although improvised principally on electric guitar, it commences with timestretched and reversed chimes (0:01) and a mellotron emulator with extensive echo to modify timbral characteristics and obscure and lengthen signal (0:20). Reversed echo sweeps (0:53) add a sense of spatial unreality, and a range of extended performance techniques are utilized as forms of auditory abstraction, such as bowing strings with a brass slide (1:10), volume swells (1:15 and 3:10), employing a violin bow (2:17) and striking strings above the nut of the guitar to produce belllike sonorities (3:44). A progressive use of frequency modulation, used in the repeated backwards guitar arpeggio (2:29) imparts both a feeling of occlusion/revealment and sensation of movement, and the slow gear effect employed on the emerging quartal motif at (3:02) modifies envelope characteristics, rendering the instrument less obviously guitar-like. The underwater sound world of "Submarine Bells" developed largely during improvisation, and although lacking more exotic instrumentation, the author felt each sound in this instance was sufficiently unusual to demonstrate many of the characteristics mentioned and achieve the desired suspended aesthetic.

\section{Conclusion}

The embodiment of the composer/instrumentalist in the music-making process should not be overlooked. Even in the fantastic worlds of psychedelia and other forms of experimental popular music, human representation is apparent despite levels of abstraction due to a corporeal recognition of spatio-temporal characteristics inherent in performance. In the artificially conceived environment of entirely electronic music and pre-recorded loops, a disconnect begins to emerge. Even in the generative soundscapes of Eno, where creative intent has been reduced almost to the role of systems manager, his authorial imprint is still evident. As Pattie and Albiez (2016) suggest, "Eno is somewhere in the system as part of the ecology of the recording, but not its focal point (...) his sensitivity to timbre (...) can't be divorced from the landscape" (2016: 2-3). The recording of "Submarine Bells" asks what a suspended aesthetic sounds like in music. Its success is dependent on a balance between adherence to the techniques listed, and a specific creative methodology that embodies the individual character of the composer.

\section{References}

\section{Bibliography}

Achtermann, M. E. 2016. Yes, but is it Music? Brian Eno and the Definition of Ambient Music. In S. Albiez and D. Pattie, Eds. Brian Eno: Oblique Music. London: Bloomsbury. 85-104.

Clarke, E. 2005. Ways of Listening: An Ecological Approach to the Perception if Musical Meaning. New York: Oxford University Press. 
Doyle, P. 2004. From 'My Blue Heaven' to 'Race with the Devil': Echo, Reverb and (dis) ordered Space in Early Popular Music Recording. Popular Music, 23(01): 31-49.

Ihde, D. 2007. Listening and Voice: Phenomenologies of Sound. 2nd ed. Albany, NY: State University of New York Press.

McClary, S. 2004. Rap, Minimalism, and Structures of Time in Late Twentieth-Century Culture. In C. Cox and D. Warner Eds. Audio Culture: Readings in Modern Music. New York: Bloomsbury. 289-298.

Pattie, D. and Albiez, S. 2016. Brian Eno: A Problem of Organization. In S. Albiez and Pattie, D. Eds. Brian Eno: Oblique Music. London: Bloomsbury. 1-8.

Reising, R. 2009. Melting Clocks and the Hallways of Always: Time in Psychedelic Music. Popular Music and Society, 32(4): 523-547.

Schaeffer, P. 2004. Acousmatics. In C. Cox and D. Warner Eds. Audio Culture: Readings in Modern Music. New York, NY: Bloomsbury Academic. 76-81

Scoates, C. 2013. Brian Eno: Visual Music. San Francisco CA: Chronicle Books.

Shouse, E. 2005. Feeling, Emotion, Affect. M/C Journal, 8(6). http://journal.mediaculture.org.au/0512/03-shouse.php. Accessed 20 October 2016.

Stern, D. N. 2010. Forms of Vitality: Exploring Dynamic Experience in Psychology, the Arts, Psychotherapy, and Development. Oxford: Oxford University Press.

Tannenbaum, R. 1985. A meeting of sound minds: John Cage and Brian Eno. Musician, September 1985. http://www.moredarkthanshark.org/eno_int_musician-sep85.html. Accessed 9 October 2016.

\section{Discography}

Eno, Brian. 1975. Discreet Music. Obscure Records, UK.

Radiohead. 2016. "Daydreaming". A Moon Shaped Pool. XL Recordings, UK.

Reich, Steve. 1968. "It's Gonna Rain". Steve Reich: Live/Electric Music. Columbia Masterworks, US.

Beatles, the. 1967. "Strawberry Fields Forever". Parlophone Records, UK.

Jimi Hendrix Experience, the. 1968. "... And the Gods Made Love". Electric Ladyland. Track Records, UK.

Pink Floyd, 1967. "See Emily Play". Columbia, UK

Small Faces, the. 1967. "Here Come The Nice". Immediate, UK.

Yardbirds, the. 1965. "Heart Full Of Soul". Columbia Records, UK.

\section{Videography}

Imaginary Landscapes. 1989. Dir. Ward, D., and Cardazzo, G. Mystic Fire Video. 